\title{
Dynamics of the reactions of muonium and deuterium atoms with vibrationally excited hydrogen molecules: tunneling and vibrational adiabaticity
}

\author{
P. G. Jambrina, ${ }^{a}$ E. García, ${ }^{b}$ V. J. Herrero, ${ }^{c}$ V. Sáez-Rábanos ${ }^{d}$ and F. J. Aoiz ${ }^{* a}$ \\ Received 25th June 2012, Accepted 3rd September 2012 \\ DOI: $10.1039 / \mathrm{c} 2 \mathrm{cp} 42130 \mathrm{e}$
}

Quantum mechanical (QM) and quasiclassical trajectory (QCT) calculations have been carried out for the exchange reactions of $\mathrm{D}$ and $\mathrm{Mu}(\mathrm{Mu}=$ muonium) with hydrogen molecules in their ground and first vibrational states. In all the cases considered, the QM rate coefficients, $k(T)$, are in very good agreement with the available experimental results. In particular, QM calculations on the most accurate potential energy surfaces (PESs) predict a rate coefficient for the $\mathrm{Mu}+\mathrm{H}_{2}$ $(\nu=1)$ reaction which is very close to the preliminary estimate of its experimental value at $300 \mathrm{~K}$. In contrast to the $\mathrm{D}+\mathrm{H}_{2}(\nu=0,1)$ and the $\mathrm{Mu}+\mathrm{H}_{2}(\nu=0)$ reactions, the QCT calculations for $\mathrm{Mu}+\mathrm{H}_{2}(\nu=1)$ predict a much smaller $k(T)$ than that obtained with the accurate QM method. This behaviour is indicative of tunneling. The QM reaction probabilities and total reactive cross sections show that the total energy thresholds for the reactions of $\mathrm{Mu}$ with $\mathrm{H}_{2}$ in $\nu=0$ and $\nu=1$ are very similar, whereas for the corresponding reaction with $\mathrm{D}$ the $\nu=0$ total energy threshold is about $0.3 \mathrm{eV}$ lower than that for $\nu=1$. The results just mentioned can be explained by considering the vibrational adiabatic potentials along the minimum energy path. The threshold for the reaction of $\mathrm{Mu}$ with $\mathrm{H}_{2}$ in both $\nu=0$ and $\nu=1$ states is the same and is given by the height of the ground vibrational adiabatic collinear potential, whereas for the $\mathrm{D}+\mathrm{H}_{2}$ reaction the adiabaticity is preserved and the threshold for the reaction in $\nu=1$ is very close to the height of the $\nu=1$ adiabatic collinear barrier. For $\mathrm{Mu}+$ $\mathrm{H}_{2}(\nu=1)$ the reaction takes place by crossing from the $\nu=1$ to the $\nu=0$ adiabat, since the exit channel leading to $\mathrm{MuH}(\nu=1)$ is not energetically accessible. At the lowest possible energies, the non-adiabatic vibrational crossing implies a strong tunneling effect through the $\nu=1$ adiabatic barrier. Absence of tunneling in the classical calculations results in a threshold that coincides with the height of the $\nu=1$ adiabatic barrier. Most interestingly, the expected tunneling effect in the reaction of $\mathrm{Mu}$ with hydrogen molecules occurs for $\mathrm{H}_{2}(\nu=1)$ but not for $\mathrm{H}_{2}(\nu=0)$ where zero-point-energy effects clearly dominate.

\section{Introduction}

The reaction $\mathrm{Mu}+\mathrm{H}_{2} \rightarrow \mathrm{MuH}+\mathrm{H}$, involving the light muonium atom - a hydrogen-like atom with a mass of $0.114 \mathrm{u}$ formed by a positive muon, $\mu^{+}$, and an electron - could be expected in principle to be an ideal system for the study of tunneling in chemical processes. However, when hydrogen molecules are in their ground internal state, a possible reactivity

${ }^{a}$ Departamento de Química Física, Facultad de Quimica, Universidad Complutense (Unidad Asociada CSIC), 28040 Madrid, Spain. E-mail:aoiz@quim.ucm.es

${ }^{b}$ Departamento de Química Física, Universidad del País Vasco (UPV/EHU), Paseo de la Universidad 7, 01006 Vitoria, Spain

${ }^{c}$ Instituto de Estructura de la Materia (CSIC), Serrano 123, 28006 Madrid, Spain.E-mail: v.herrero@csic.es

${ }^{d}$ Departamento de Química y Bioquímica, ETS Ingenieros de Montes, Universidad Politécnica, 28040 Madrid, Spain enhancement due to tunneling has been found ${ }^{1-3}$ to be largely superseded by the effect of the high zero point energy (ZPE) and therefore of a large endoergicity, which leads to an appreciable growth in the reaction threshold and consequently to a notable decrease in the value of the rate coefficient as compared with that of the heavier isotopologues of $\mathrm{H}+\mathrm{H}_{2}{ }^{4}$ This fact results in a very large inverse kinetic isotope effect (KIE) that has been recently highlighted ${ }^{1,5}$ through the comparison of the rate coefficients for the two extreme isotopic variants of the reaction; namely, $\mathrm{Mu}+\mathrm{H}_{2}$ and $\mathrm{He} \mu+\mathrm{H}_{2}$, where $\mathrm{He} \mu$ with a mass of $4.1155 \mathrm{u}$ is muonic helium, ${ }^{5} \mathrm{a} \mathrm{He}$ atom in which one of the electrons has been substituted by a negative muon, $\mu^{-}$.

In an analysis based on variational transition state theory (VTST) Fleming et al. ${ }^{1,5}$ attributed the pronounced $\mathrm{Mu} / \mathrm{He} \mu$ KIE to the difference in the stretching vibration ZPE of the two reactions at the transition state. The good agreement 
between the VTST and experimental KIE was invoked by these authors in support of the existence of quantized transition states ${ }^{6,7}$ that would act as effective reaction barriers. In a recent work, Jambrina et $a .^{2}$ noted that the marked KIE observed between the reactions of $\mathrm{Mu}$ and $\mathrm{He} \mu$ (or, more generally, between $\mathrm{Mu}$ and the rest of the $\mathrm{H}$ isotopic variants) could also be obtained with quasiclassical trajectories (QCT) by using a Gaussian binning (GB) filter ${ }^{2,8,9}$ which effectively selects trajectories with an action close to that of the actual ground state of the $\mathrm{MuH}$ product molecule. Although the QCT method does not contemplate the quantization of the transition state, it imposes a product channel condition that favours vibrationally adiabatic trajectories and provides a sort of classical analog of the quantized transition states. In the case of $\mathrm{Mu}$, a large degree of vibrational adiabaticity is imposed with the GB procedure, but even without any bias in the exit channel, QCT calculations for the $\mathrm{H}+\mathrm{H}_{2}$ reaction tend to preserve a significant fraction of the reactants' vibrational energy as the reaction unfolds. ${ }^{10,11}$

The possibility of analyzing in more detail the effects of tunneling, quantized transition states, or vibrational adiabaticity on the reactive dynamics of $\mathrm{Mu}+\mathrm{H}_{2}$ is certainly attractive. However, for the reaction with ground vibrational state $\mathrm{H}_{2}$ molecules, any manifestation of these effects is likely to be blurred by the large influence of the ZPE, as has been recently corroborated $^{3}$ using the Ring Polymer Molecular Dynamics (RPMD) method. To overcome this limitation we have undertaken in this work a quantum mechanical and QCT study of the reactivity of $\mathrm{Mu}+\mathrm{H}_{2}(\nu=1)$. This slightly exoergic process $(\sim 0.20 \mathrm{eV})$ is not constrained by the high ZPE of MuH in the exit channel and provides a unique occasion for the investigation of detailed aspects of the dynamics of a reaction involving the lightest and the presumably more "quantal" hydrogen isotope. The results are compared to the recent preliminary estimate of the $\nu=1$ rate coefficient for this reaction at $300 \mathrm{~K} .{ }^{12}$ We have also performed parallel calculations for the $\mathrm{D}+\mathrm{H}_{2}(\nu=1)$ isotopic variant, where pure quantum mechanical effects are expected to be smaller considering the big disparity in the reduced masses of the two systems $\left(\mu_{\mathrm{D}+\mathrm{H}_{2}} / \mu_{\mathrm{Mu}+\mathrm{H}_{2}}=9.337\right)$.

The paper is organized as follows: Section II contains a brief account of the theoretical methods employed. In Section III the results of the calculations are analyzed and discussed. Finally, a summary and conclusions are presented in Section IV.

\section{Theoretical method}

Time independent quantum mechanical (QM) and QCT calculations were carried out on the BKMP2 PES of Boothroyd et al. ${ }^{13}$ to obtain thermal rate coefficients for the $\mathrm{Mu}+\mathrm{H}_{2}$ $(\nu=1)$ and $\mathrm{D}+\mathrm{H}_{2}(\nu=1)$ reactions in the temperature range between 200 and $1000 \mathrm{~K}$. A description of the QM and QCT theoretical methods can be found in ref. 14 and 15, respectively; therefore, here we will only mention the details of the current calculations.

The QCT $k(T)$ was obtained following the Cumulative Reaction Probability (CRP) formulation described in ref. 15 and 16. This procedure has been previously used in ref. 2 to compute thermal rate coefficients for the $\mathrm{Mu}+\mathrm{H}_{2}$ reaction. For $\mathrm{D}+\mathrm{H}_{2}$, a batch of $3 \times 10^{7}$ trajectories was run in the
$0.5-1.6 \mathrm{eV}$ total energy range. Similarly, $1.8 \times 10^{7}$ trajectories were run for the $\mathrm{Mu}+\mathrm{H}_{2}(\nu=1)$ reaction.

For the assignment of final states to each trajectory two methods have been used. The standard assignment is the histogram-binning, hereinafter QCT-HB, that consists of rounding the classical product's vibrational quantum number to the nearest integer; hence it gives the same weight to all trajectories, regardless of their vibrational or rotational action. The drawback of this method is that it allows the population of states which are not energetically accessible. To avoid this problem, a second procedure is used, the Gaussian-binning (QCT-GB) method ${ }^{2,8,9}$ that consists of weighting each trajectory according to the value of a Gaussian distribution centered on the right (integer) value of the QM vibrational quantum number of the products, such that the closer is the classical action to the QM one, the larger is its weight. The QCT-HB method tends to overestimate the reactivity of endoergic reactions with a large product's ZPE. In contrast, the QCT-GB procedure overcomes this problem since the weight applied to those trajectories whose vibrational action is well below the ZPE is practically null and contribute very little to the cross section. As a result of that, the reactivity of endoergic reactions (with a large product's ZPE) is considerably smaller and in most instances agrees much better with the exact $\mathrm{QM}$ results. In all cases studied here, we have used a full-width-half-maximum $\Delta \nu=0.1$ for the Gaussian distribution. The rate coefficients for the $\mathrm{D}+\mathrm{H}_{2}(\nu=0,1)$ are largely insensitive to the specific value of this width. However, as discussed in ref. 2, the values of $k(T)$ for the $\mathrm{Mu}+\mathrm{H}_{2}(\nu=0)$ are strongly affected by this value, this being one of the most serious limitations of the GB procedure.

The QM calculations have been carried out using the coupledchannel hyperspherical coordinate method implemented in the $\mathrm{ABC}$ code of Skouteris et al. ${ }^{14}$ For the $\mathrm{Mu}+\mathrm{H}_{2}$ reaction, a grid of 280 total energies was employed spanning the $0.5 \mathrm{eV}$ to $2.0 \mathrm{eV}$ total energy range. The basis included all the diatomic levels up to a cutoff energy of $2.85 \mathrm{eV}$ and comprised all the helicity quantum numbers, $\Omega$. All partial waves up to $J_{\max }=25$ were considered and the log-derivative propagation was extended in 250 sectors up to $25 a_{0}$. For $\mathrm{D}+\mathrm{H}_{2}$, a grid of 320 total energies between $0.3 \mathrm{eV}$ and $1.8 \mathrm{eV}$ was employed. The basis included all the diatomic levels up to $2.85 \mathrm{eV}$. A maximum value of the helicity quantum number of $\Omega_{\max }=6$ was enough to ensure convergence. Partial waves up to $J_{\max }=40$ had to be included and the log-derivative propagation was done in 150 sectors up to $18 a_{0}$.

Once the CRP has been obtained by using the methodology described above, the expression of the thermal rate coefficients can be written as: ${ }^{15}$

$$
k(T)=\frac{\int_{0}^{\infty} C_{r}(E) \exp \left(-E / k_{\mathrm{B}} T\right) \mathrm{d} E}{h \Phi_{\mathrm{rel}}(T) Q_{v, j}^{\mathrm{H}_{2}}(T)}
$$

where the total CRP for a homonuclear molecule as a function of the total energy, $E$, is given by

$$
C_{r}(E)=\sum_{T}(2 \mathrm{~T}+1) \sum_{J=0}^{J_{\max }}(2 J+1) C_{r}^{J, \mathrm{~T}}(E)
$$

where $\mathrm{T}$ is the molecular nuclear spin quantum number. For the $\mathrm{H}_{2}$ molecule, where the atomic nuclear spin $I=1 / 2, \mathrm{~T}=0$ for para-hydrogen (even rotational states) and $\mathrm{T}=1$ for 
ortho-hydrogen (odd rotational states). The CRP for a given value of the total angular momentum and diatomic parity is $C_{r}^{J, T}(E)$. In eqn (1), $k_{\mathrm{B}}$ is the Boltzmann constant, and $\Phi_{\mathrm{rel}}(T)$ and $Q_{v, j}^{\mathrm{H}_{2}}(T)$ are the translational and coupled nuclear-rovibrational partition functions, respectively. In eqn (1) and (2) it is implicitly assumed that there is ortho-para equilibrium at any temperature.

For the purposes of this work, it is also interesting to define the thermal-CRP including the reagent's partition function as:

$$
C_{r}(E ; T)=\frac{C_{r}(E) \exp \left(-E / k_{\mathrm{B}} T\right)}{h \Phi_{\mathrm{rel}}(T) Q_{v, j}^{\mathrm{H}_{2}}(T)}
$$

whose integration over the total energy yields the $k(T)$. The thermal-CRP represents the contribution of the Boltzmann weighted CRP to the rate coefficient in an interval of energies within $E$ and $E+\mathrm{d} E$.

As mentioned above, the present calculations were mostly carried out on the electronically adiabatic BKMP2 PES. A more precise evaluation of the rate coefficients, especially for the $\mathrm{Mu}+\mathrm{H}_{2}$ reaction, requires the inclusion of the mass dependent diagonal correction to the Born-Oppenheimer (BO) potential. In order to account for this effect, following ref. 17 we have multiplied the $\mathrm{BO}$ QM and QCT $k(T)$ calculated on the BKMP2 PES by an additional Boltzmann factor, $\exp \left(-\Delta E_{\mathrm{b}} / k_{\mathrm{B}} T\right)$, where the mass dependent $\Delta E_{\mathrm{b}}$ is the increase in the barrier due to the aforementioned non-BO diagonal term, which is obviously different for the various isotopologues of $\mathrm{H}_{2}$. In our previous work, ${ }^{2}$ the value of $\Delta E_{\mathrm{b}}$ was taken as the difference between the saddle points in the minimum energy path (MEP) of the BO BKMP2 and the Born-Huang (BH) PES by Mielke et al., ${ }^{18}$ which includes the adiabatic diagonal term. In the case of the $\mathrm{Mu}+\mathrm{H}_{2}$ reaction that value is $16.04 \mathrm{meV}$. In the present work, however, following the suggestion of ref. 5 (see, however, ref. 19) we have calculated $\Delta E_{\mathrm{b}}$ as the difference between the barrier heights of the respective $\nu=0$ adiabatic collinear potential of the two PESs. The barrier heights were computed using the ABCRATE $\operatorname{code}^{20}$ and the following values of $\Delta E_{\mathrm{b}}$ were obtained: $10.36 \mathrm{meV}$ for the $\mathrm{Mu}+\mathrm{H}_{2}$ and $6.11 \mathrm{meV}$ for the $\mathrm{D}+\mathrm{H}_{2}$ reaction. The differences in the values of $k(T)$ with and without the diagonal adiabatic correction are in general small for $T>200 \mathrm{~K}$. The largest differences occur for the $\mathrm{Mu}+\mathrm{H}_{2}$ reaction; at $300 \mathrm{~K}$ the $\mathrm{QM}$ thermal $(\nu=0) k(T)$ are $5.521 \times 10^{-20}$ and $8.242 \times 10^{-20} \mathrm{~cm}^{3} \mathrm{~s}^{-1}$, with and without the adiabatic correction respectively.

To test the validity of this correction the QM thermal rate coefficients for the $\mathrm{Mu}+\mathrm{H}_{2}$ reaction were also computed on the $\mathrm{BH}$ PES by running 300 energies in the $0.59-2.1 \mathrm{eV}$ total energy range. The cutoff energy was set to $3 \mathrm{eV}$ and the basis included all the helicities up to 6 . The propagation was performed in 300 sectors up to $R_{\max }=18 a_{0}$.

Strictly speaking, once a reaction channel is open (i.e., energetically accessible), there is no QM threshold in the reaction probability, even in the presence of a barrier, due to the contribution of tunneling. However, for the purposes of the present work it is convenient to define an operational QM threshold in order to compare QM and QCT results. This operational threshold is here defined as the total energy at which the reaction probability, $P^{J}(E)$, for $J=0$ takes the value of $10^{-3}$. Other criteria based on the values of the thermal CRP lead to similar values.

\section{Results and discussion}

The QM and QCT rate coefficients for the reactions of $\mathrm{Mu}$ with thermal $\mathrm{H}_{2}$ (mostly in $\left.\nu=0\right)$ and with $\mathrm{H}_{2}(\nu=1)$ are displayed in the upper and lower panels, respectively, of Fig. 1. For the reaction with thermal $\mathrm{H}_{2}$ (upper panel) the QM rate coefficients are in very good agreement with the available experimental measurements ${ }^{21}$ and also in better agreement than previous accurate $\mathrm{QM}$ calculations on a previous PES. $^{22,23}$ The QCT-GB calculations, which weight preferentially trajectories with a vibrational action close to the actual $\nu=0$ state of $\mathrm{MuH}$, perform also reasonably well, albeit somewhat worse than their QM counterparts. In contrast, the QCT-HB results, that ignore altogether the quantization of the products, yield too high rate coefficients because they include a large contribution of unphysical trajectories with products' vibrational energies below the $\mathrm{ZPE}$ of $\mathrm{MuH}$.

The situation is, however, quite different for the reaction of hydrogen molecules excited to their first vibrational state as shown in the bottom panel of Fig. 1. As expected, the rate coefficients are much larger than those for the reaction with ground state $\mathrm{H}_{2}$ (as much as seven orders of magnitude
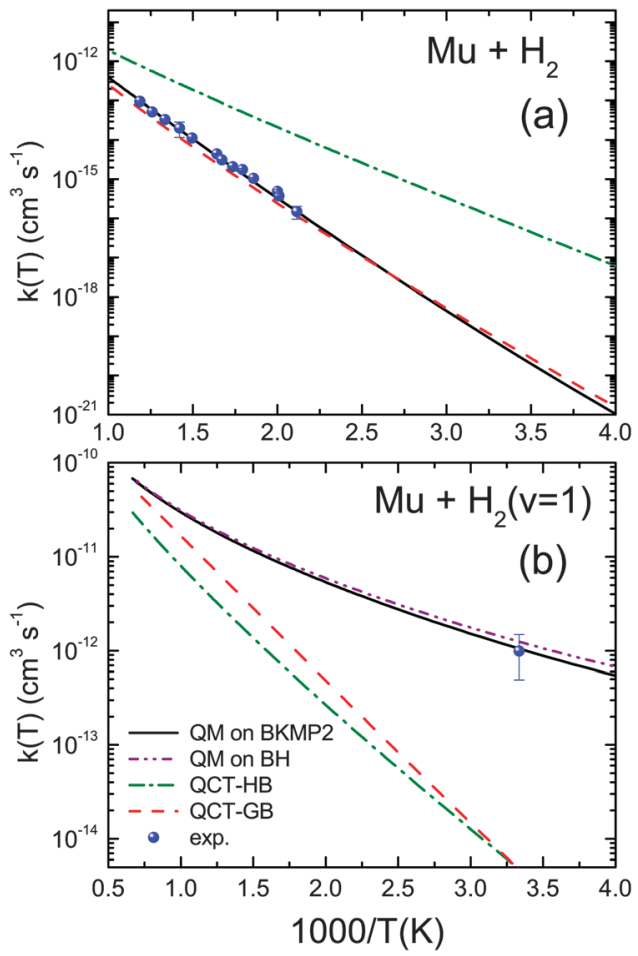

Fig. 1 Rate coefficients $\left(\mathrm{cm}^{3} \mathrm{~s}^{-1}\right)$ for: (a) thermal $\mathrm{Mu}+\mathrm{H}_{2}$. Blue solid circles: experimental data from ref. 21. Black solid line: QM calculations on BKMP2 PES. Dashed red line: QCT-GB calculations on the BKMP2 PES. Dash-dotted green line: QCT-HB calculations on the BKMP2 PES. (b) $\mathrm{Mu}+\mathrm{H}_{2}(\nu=1)$. Solid blue circle: experimental value. ${ }^{12}$ Black solid line: QM calculations on BKMP2 PES. Purple dash-dot-dotted line: present calculations on the BH PES. ${ }^{18}$ Rest of the lines as in panel (a). In all the calculations on the BKMP2 PES the adiabatic diagonal correction has been included (see the text). 
at $T=300 \mathrm{~K}$ ), but in this case the two QCT approaches show a similar behaviour and diverge largely with decreasing temperature from the corresponding quantum rate coefficients which take much higher values. The two sets of quantum results represented in the lower panel of Fig. 1 correspond to calculations carried out on both the BKMP2 surface ${ }^{13}$ including the non-BO diagonal correction (i.e., the adiabatic approximation) as indicated in the previous section, and in the recent highly accurate Born-Huang PES by Mielke et al. ${ }^{18}$ in which the diagonal correction is already built in.

The recent estimate of the experimental rate coefficient ${ }^{12}$ is in good agreement with the QM results of both PESs and is larger by more than two orders of magnitude than the rate coefficients from the QCT calculations at the same temperature (see Table 1 for a detailed comparison). The departure of the QM rate coefficients from the linear Arrhenius behaviour is evident in the evolution of $k(T)$ with decreasing temperature (bottom panel of Fig. 1) and is clearly suggestive of tunneling.

Analogous results for the $\mathrm{D}+\mathrm{H}_{2}$ isotopologue are shown in Fig. 2. As in the previous case, the upper panel of this figure corresponds to the reaction with thermal $\mathrm{H}_{2}$ molecules (essentially in $\nu=0$ ), and the lower panel to $\mathrm{H}_{2}$ molecules excited to the first vibrational level. It is clear at first sight that for this isotopic variant the results of the two QCT methods and of the QM calculations are by far not so discrepant as those just described for the reaction with $\mathrm{Mu}$. The rate coefficient measurements for the reaction with thermal $\mathrm{H}_{2}$ molecules extend over five orders of magnitude (see ref. 17, 24 and references therein) and are very well reproduced by the QM calculations. The QCT-HB values give the right trend, but are systematically lower than the QM and experimental data. In turn, rather interestingly, the QCT-GB method predicts larger rate coefficients which are in a global agreement with the measurements. For the reaction with $\mathrm{H}_{2}(\nu=1)$ the ultimate experimental datum $\left((2.1 \pm 0.6) \times 10^{-13} \mathrm{~cm}^{3} \mathrm{~s}^{-1}\right),{ }^{25}$ corresponding to $T=330 \mathrm{~K}$, is in reasonable agreement with the $\mathrm{QM}$ and QCT theoretical calculations, below this temperature the differences between QM and QCT rate coefficients increase, with the QM $k(T)$ becoming gradually larger than its QCT counterparts, and showing an upward curvature indicative of a likely tunneling contribution to the reactivity. At $T=200 \mathrm{~K}$, the QM rate coefficient is larger by a factor of two than that from the QCT-GB method.

The QM and QCT kinetic isotope effect (KIE) for the reactions of $\mathrm{Mu}$ and $\mathrm{D}$ with $\mathrm{H}_{2}(\nu=1)$; i.e., the ratio of their respective rate coefficients as a function of temperature, is displayed in Fig. 3 together with an approximate experimental KIE at $T \sim 300 \mathrm{~K}$. This experimental KIE has been derived as the quotient between the rate coefficient values of $\mathrm{Mu}+\mathrm{H}_{2}$ $(\nu=1)$ estimated at $300 \mathrm{~K},{ }^{12}$ and that of $\mathrm{D}+\mathrm{H}_{2}(\nu=1)^{25}$ which corresponds to $330 \mathrm{~K}$. Although the temperature of the two experiments is not the same, the difference is small enough
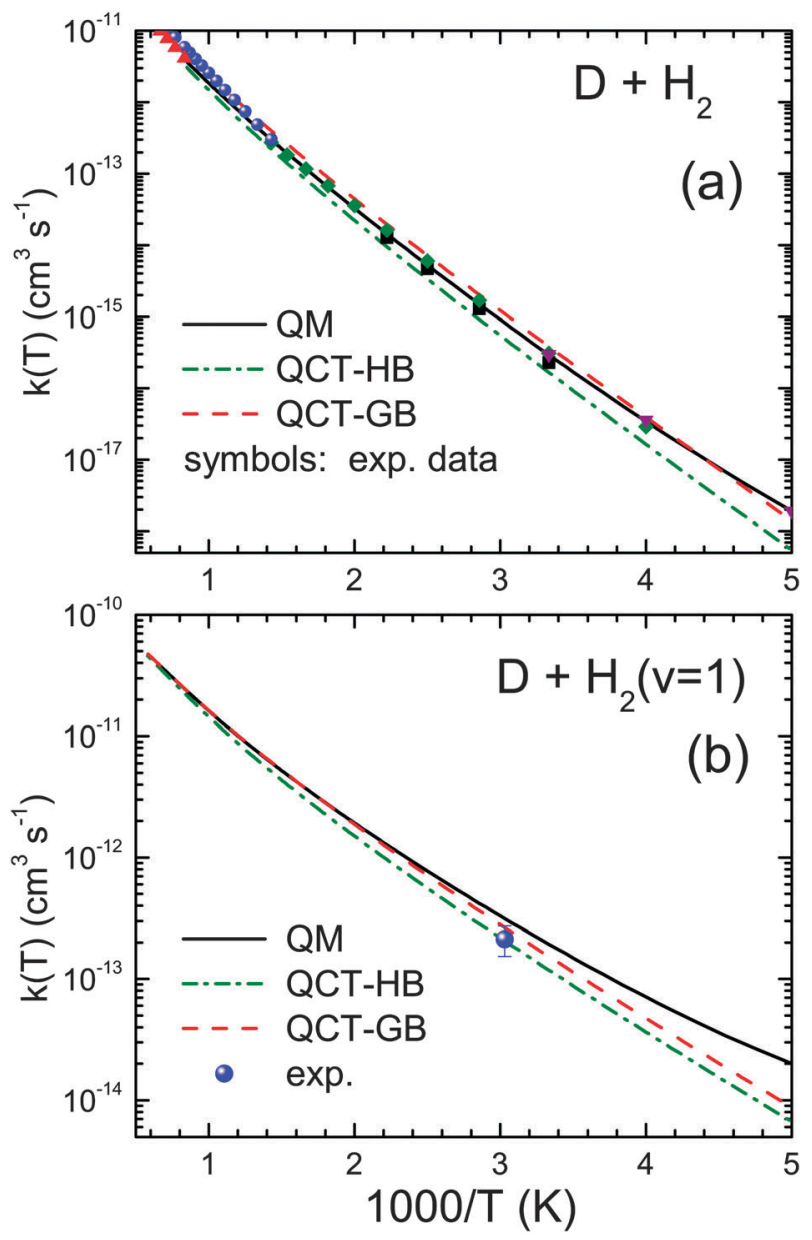

Fig. 2 Rate coefficients $\left(\mathrm{cm}^{3} \mathrm{~s}^{-1}\right)$ for: (a) thermal $\mathrm{D}+\mathrm{H}_{2}$. Symbols: experimental data from the Arrhenius parameters given in ref. 17 and 24. Black solid line: QM results. Dashed red line: QCT-GB calculations. Dash-dotted green line: QCT-HB calculations. (b) $\mathrm{D}+\mathrm{H}_{2}(\nu=1)$ solid blue circle: experimental datum from ref. 25. Rest of the lines as in panel (a). All the calculations have been performed on the BKMP2 PES and include the adiabatic diagonal correction (see the text).

for the KIE to be meaningful considering that the error in the KIE is larger than $50 \%$ - mainly caused by the uncertainty in the value of the $k(T)$ in the $\mathrm{Mu}+\mathrm{H}_{2}(\nu=1)$ reaction. The QM KIE, which reproduces very well the experimental point, is always higher than one, reflecting the larger values of the $k(T)$ of the $\mathrm{Mu}+\mathrm{H}_{2}(\nu=1)$ reaction as compared to those for $\mathrm{D}+\mathrm{H}_{2}(\nu=1)$, calculated including the respective diagonal corrections but otherwise on an identical PES. This fact stresses further the likelihood of appreciable tunneling in the reaction of $\mathrm{Mu}$ with $\mathrm{H}_{2}(\nu=1)$ which otherwise is absent in the reaction with $\mathrm{H}_{2}(\nu=0)$. The QCT method, unable to account for possible tunneling effects, yields always values of the KIE lower than one, which can easily be traced back to the

Table 1 Rate coefficients at $T=300 \mathrm{~K}$ for the $\mathrm{Mu}+\mathrm{H}_{2}(\nu=1) \rightarrow \mathrm{MuH}+\mathrm{H}$ reaction calculated by the different theoretical approaches in comparison with the experimental estimate. ${ }^{12}$ All the values are given in $\mathrm{cm}^{3} \mathrm{~s}^{-1}$. The calculations on the BKMP2 PES include the adiabatic diagonal correction (see the text for more details)

\begin{tabular}{llllll}
\hline$T$ & Exp & QM-BKMP2 & QM-BH & QCT-HB & QCT-GB \\
\hline $300 \mathrm{~K}$ & $(1.0 \pm 0.5) \times 10^{-12}$ & $1.05 \times 10^{-12}$ & $1.25 \times 10^{-12}$ & $4.76 \times 10^{-15}$ & $4.72 \times 10^{-15}$ \\
\hline
\end{tabular}




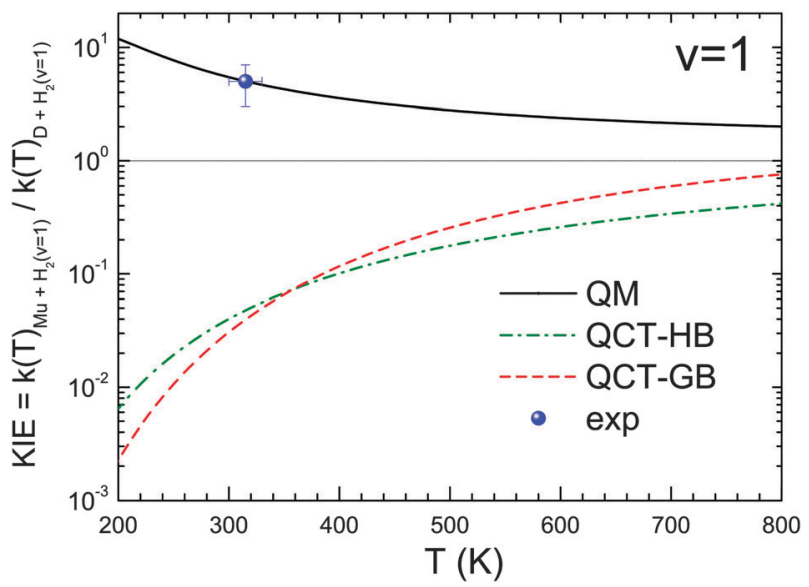

Fig. 3 Kinetic isotope effect (KIE) $k(T)_{\mathrm{Mu}+\mathrm{H}_{2}} / k(T)_{\mathrm{D}+\mathrm{H}_{2}}$. Blue circle: experimental datum. The horizontal bar stresses the fact the temperature at which the respective rate coefficients are not the same (see the text for details). Lines as in Fig. 2.

very low QCT rate coefficients for the reaction of $\mathrm{Mu}$ with $\mathrm{H}_{2}$ $(\nu=1)$ commented on above. It is also worth noting at this point that the $\mathrm{Mu} / \mathrm{He} \mu$ or $\mathrm{Mu} / \mathrm{D}$ KIE for the reaction with $\mathrm{H}_{2}$ $(\nu=0)$ is also significantly lower than one $\left(\approx 1 \times 10^{-2}\right)^{1,2,5}$ due to the already mentioned predominance of ZPE effects over any possible tunneling in the reaction of ground state $\mathrm{H}_{2}$ with $\mathrm{Mu}$.

Fig. 4 shows the thermal CRPs at $500 \mathrm{~K}$ for the reactions of $\mathrm{Mu}$ and $\mathrm{D}$ with $\mathrm{H}_{2}(\nu=1)$. This kind of plot shows in a clear way the evolution of reactivity as a function of total energy for a given $T$ (in fact the thermal rate coefficient is the integral of the represented curves). The different reactive behaviour predicted by the QM and QCT methods for $\mathrm{Mu}+\mathrm{H}_{2}(\nu=1)$ is made immediately apparent in this representation. The QM operational threshold (see previous section for a definition) is considerably lower $(\approx 0.8 \mathrm{eV})$ than the QCT one $(\approx 1.05 \mathrm{eV})$ and, in fact, the maximum in the QM thermal CRP occurs at a total energy below the QCT threshold, which stresses further the likely huge contribution of tunneling to the values of the rate coefficient advanced above. At lower temperatures,

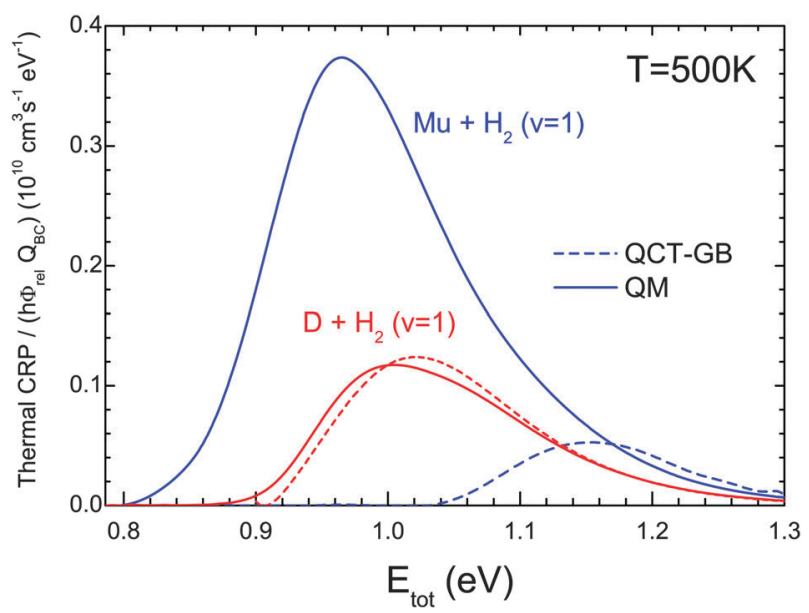

Fig. 4 QM (continuous lines) and QCT (dashed lines) thermal CRP at $T=500 \mathrm{~K}$ for the $\mathrm{Mu}+\mathrm{H}_{2}(\nu=1)$ (blue lines) and for the $\mathrm{D}+\mathrm{H}_{2}$ $(\nu=1)$ (red lines). the ratio between the QM and QCT thermal CRPs is even larger, and the latter appears as a minuscule contribution at the highest energies. Actually, as shown in Fig. 4, even at $500 \mathrm{~K}$ the thermal QCT CRP accounts only for a small highenergy fraction of the corresponding QM one. In contrast, the QM and QCT thermal CRPs for D $+\mathrm{H}_{2}(\nu=1)$ are comparatively similar and lie between the two CRPs of $\mathrm{Mu}$. A close inspection reveals that also in this case the QM thermal CRP has a lower threshold and takes higher values towards the low energy end, which is indicative of a certain tunneling contribution. Although this effect is much smaller than in the case of $\mathrm{Mu}$, it has also an influence on the corresponding rate coefficient below $400 \mathrm{~K}$.

The evolution of the QM and QCT cross sections as a function of the total energy can be compared for the reactions of $\mathrm{Mu}$ and $\mathrm{D}$ with $\mathrm{H}_{2}(\nu=0)$ and $\mathrm{H}_{2}(\nu=1)$ in Fig. 5. The comparison is in all cases restricted to the ground rotational state $(j=0)$ of the $\mathrm{H}_{2}$ molecule. As expected from the rate coefficient results commented on thus far, there is a reasonable agreement between QM and QCT calculations for the reactions with $\mathrm{D}$ and there are noteworthy discrepancies in the reactions with $\mathrm{Mu}$. The most obvious differences, associated with limitations of the QCT methods, are worth commenting on briefly. In the $\mathrm{Mu}+\mathrm{H}_{2}(\nu=0)$ reaction (upper left panel), controlled by ZPE effects, the neglect of the zero point energy of the products inherent to the QCT-HB binning is apparent in the artificially low reaction total energy threshold, $E_{0}$, obtained with this procedure. In the $\mathrm{Mu}+\mathrm{H}_{2}(\nu=1)$ reaction (lower left panel), which is not limited by ZPE effects, the two classical methods lead to a threshold $(\approx 1.05 \mathrm{eV})$ higher than the operational threshold $(\sim 0.80 \mathrm{eV})$ derived from QM calculations, hinting again at tunneling. Finally, the pronounced bumps observed in the QCT-GB results for the two reactions with $\mathrm{Mu}$ are ultimately due to the somewhat anomalous classical vibrational energy distributions that favour reactive trajectories close to the actual vibrational energy of the product molecule. In the reactions with deuterium the distortion caused by the Gaussian binning is much smaller, but still appreciable in the form of small shoulders. The most interesting feature reflected in Fig. 5 is, however, the different threshold behaviour of $\nu=0$ and $\nu=1$ in the two isotopic variants. Whereas the reactions of $\mathrm{Mu}$ with $\mathrm{H}_{2}(\nu=0)$ and $\mathrm{H}_{2}(\nu=1)$ have practically the same QM (operational) total energy threshold $\left(E_{0} \sim 0.80\right.$ and $0.84 \mathrm{eV}$ respectively), the corresponding values for the reactions with $\mathrm{D}$ differ significantly: $E_{0} \sim 0.52 \mathrm{eV}$ for $\mathrm{D}+\mathrm{H}_{2}(\nu=0)$, and $E_{0} \sim 0.87 \mathrm{eV}$ for $\mathrm{D}+\mathrm{H}_{2}(\nu=1)$.

In order to gain more insight into the causes of the distinct threshold behaviour just commented on, we have analyzed the relationship between the reaction probabilities and relevant potential energy profiles of the two isotopic variants. The results of this analysis are presented in Fig. 6 and 7.

Fig. 6 corresponds to the $\mathrm{Mu}+\mathrm{H}_{2}$ system. The left panel contains the minimum energy path on the electronic PES (lowest curve) as well as the adiabatic energy paths for $\mathrm{H}_{2}$ ( $\nu=0)$ and $\mathrm{H}_{2}(\nu=1)$, both in three dimensional (3D) (symmetric stretching + bending) and in collinear configurations (only considering the symmetric stretching). The natural mass scaled coordinate, $s$, is used for this representation. 

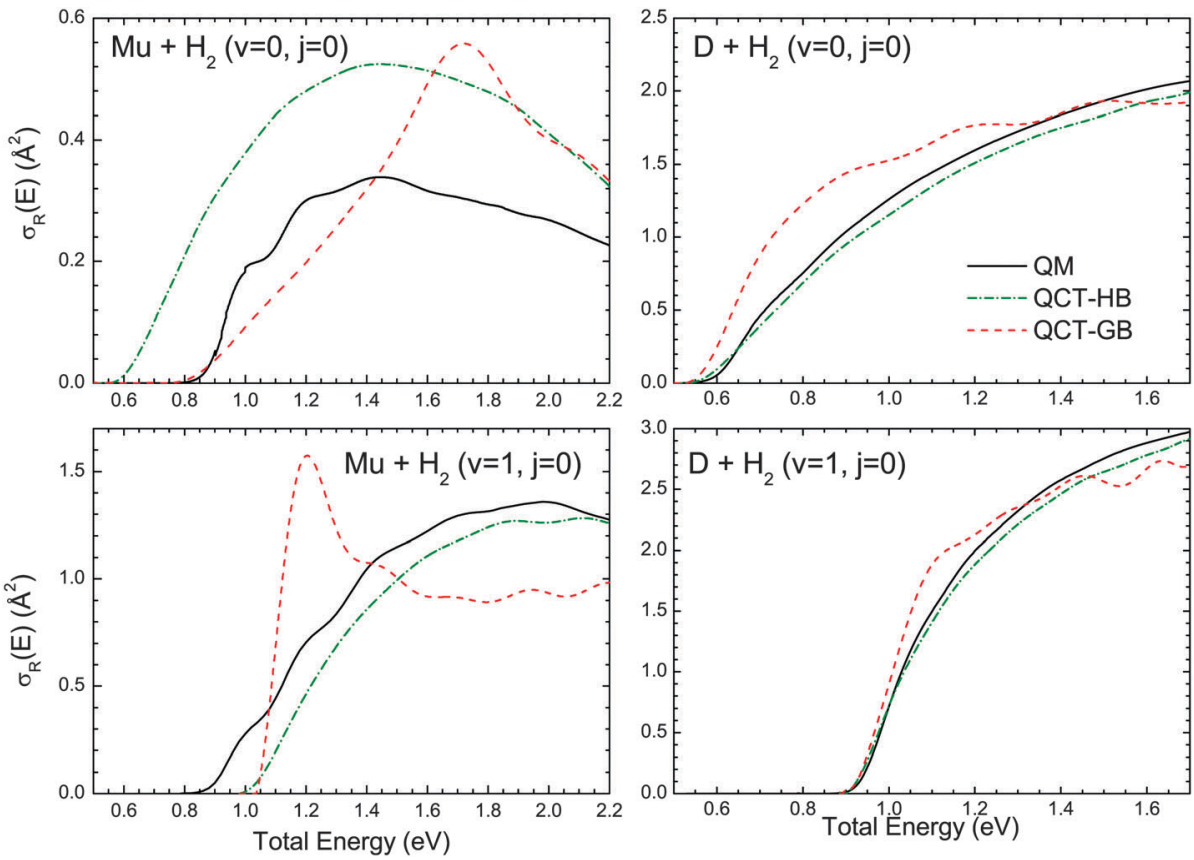

Fig. 5 Total reaction cross section $\left(\AA^{2}\right)$ as a function of the total energy, $\sigma_{\mathrm{R}}(E)$, for the indicated reactions and initial states. Lines as in Fig. 1. Note that the QM total energy threshold is very similar for the reaction of $\mathrm{Mu}$ with $\mathrm{H}_{2}(\nu=0)$ and $\mathrm{H}_{2}(\nu=1)$, whereas it is conspicuously different for the respective reactions with $\mathrm{D}$.

The coordinate $s$ is a measure of the progress of the reaction and is calculated as the distance from the saddle point. In terms of the mass scaled coordinates $X$ and $Y$ the modulus of $s$ is defined as: ${ }^{20}$

$$
|s|=\sqrt{\left(X-X^{\mathrm{SP}}\right)^{2}+\left(Y-Y^{\mathrm{SP}}\right)^{2}}
$$

while the mass scaled coordinates are related to the internuclear distances for an $\mathrm{A}+\mathrm{BC} \rightarrow \mathrm{AB}+\mathrm{C}$ reaction as follows:

$$
\begin{gathered}
X=R_{\mathrm{AB}}+\frac{m_{\mathrm{C}}}{m_{\mathrm{BC}}} R_{\mathrm{BC}} \\
Y=R_{\mathrm{BC}} \sqrt{\frac{\mu_{\mathrm{BC}}}{\mu_{\mathrm{A}-\mathrm{BC}}}}
\end{gathered}
$$

where $\mu_{\mathrm{BC}}$ and $\mu_{\mathrm{A}-\mathrm{BC}}$ are the reduced masses of the reactant diatom and triatom, respectively; $m_{\mathrm{C}}$ and $m_{\mathrm{BC}}$ are the masses of the $\mathrm{C}$ atom and $\mathrm{BC}$ diatom, respectively. $R_{\mathrm{AB}}$ and $R_{\mathrm{BC}}$ are the $\mathrm{AB}$ and $\mathrm{BC}$ internuclear distances. In eqn (4), $X^{\mathrm{SP}}$ and $Y^{\mathrm{SP}}$

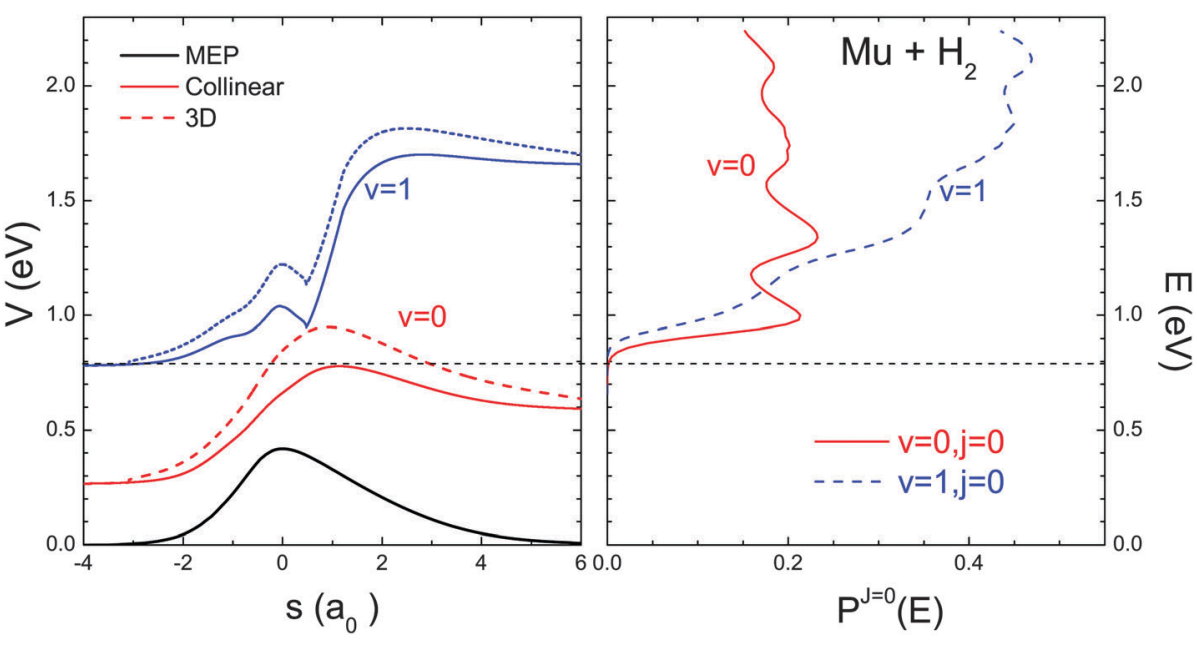

Fig. 6 Left panel: minimum energy path (MEP) (solid black line) and vibrationally adiabatic potentials along the MEP for the $\mathrm{Mu}+\mathrm{H}_{2}(\nu=0,1)$ reaction. Solid red line: collinear (only stretching contribution) $\nu=0$ adiabat. Dashed red line: three dimensional (including the bending contribution) $\nu=0$ adiabat. Solid blue line: collinear (only stretching contribution) $\nu=1$ adiabat. Short-dashed blue line: three dimensional (including the bending contribution) $\nu=1$ adiabat. Right panel: $J=0$ total reaction probability as a function of the total energy for the $\mathrm{Mu}+\mathrm{H}_{2}(\nu=0)($ red continuous line) and $\mathrm{Mu}+\mathrm{H}_{2}(\nu=1)$ (blue dashed line). The horizontal dash line highlights the correspondence between the maximum of the $\nu=0$ collinear adiabatic barrier and the 'operational' total energy threshold for the reactions with $\mathrm{H}_{2}(\nu=0)$ and $\mathrm{H}_{2}(\nu=1)$. 


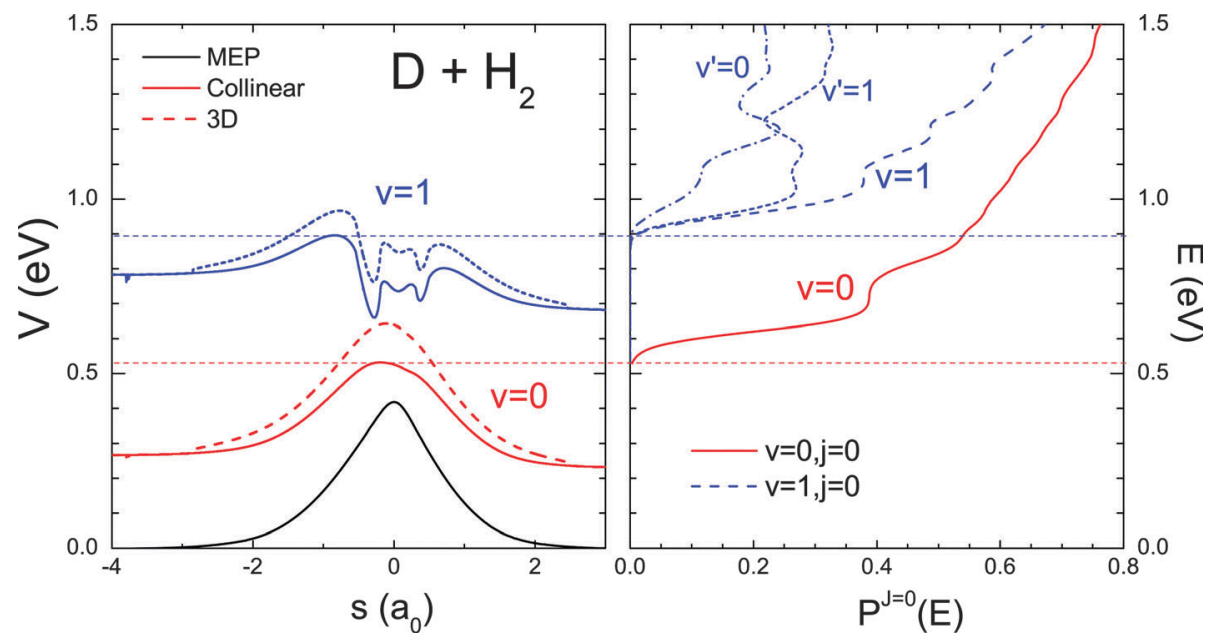

Fig. 7 The same as Fig. 6 but for the $\mathrm{D}+\mathrm{H}_{2}(\nu=0,1)$ reactions. The $\nu^{\prime}=0$ and $\nu^{\prime}=1$ contributions to the total reaction probability for $\mathrm{H}_{2}$ $(\nu=1)$ are also shown. The red and blue horizontal lines connect the top of the $\nu=0$ and $\nu=1$ collinear adiabatic barriers with the reaction probability thresholds for the two vibrational states.

are the values of the mass scaled coordinates that define the saddle point. Finally the sign of $s$ is chosen arbitrarily, and positive/negative values of $s$ are used for the description of the product/reactant valleys.

The right panel displays the value of the QM $J=0$ reaction probabilities, $P^{J=0}(E)$, for the reaction of $\mathrm{Mu}$ with $\mathrm{H}_{2}$ in $\nu=0$ and $\nu=1$. Inspection of Fig. 6 shows that for $\mathrm{Mu}+\mathrm{H}_{2}(\nu=0)$ the operational reaction threshold $(\approx 0.79 \mathrm{eV})$ nearly coincides with the maximum of the corresponding collinear adiabatic energy path $(0.78 \mathrm{eV})$, a fact that points to the absence of tunneling through the collinear vibrationally adiabatic barrier, and is consistent with the dominance of ZPE constraints commented above and in previous publications. ${ }^{2,3,26}$ As indicated elsewhere ${ }^{2,10,11}$ there seems to be a strong tendency to conserve the symmetric stretching motion in the course of the reactive encounter or, in other words, the energy deposited into this mode does not help much to overcome the barrier on the electronic PES. It is also worth noting that the location of the first maximum in $P^{J=0}(E)$ for $\nu=0$, associated with the first dynamical bottleneck, ${ }^{6,27}$ corresponds roughly with the $3 \mathrm{D}$ adiabatic barrier for the reaction which includes the bending motion. For $\mathrm{Mu}+\mathrm{H}_{2}(\nu=1)$, the operational threshold is only slightly larger $(\equiv 0.83 \mathrm{eV})$ than that for $\mathrm{Mu}+\mathrm{H}_{2}(\nu=0)$. In the post threshold region of the reaction with vibrationally excited hydrogen molecules, the reactants, which start on the adiabatic path for $\nu=1$, must cross to the $\nu=0$ path because the vibrationally adiabatic exit channel, leading to $\mathrm{MuH}(\nu=1)$, opens at much higher energy (above $1.5 \mathrm{eV}$ ). The observed threshold implies that "tunneling" through the $\nu=1$ adiabatic curve breaks the vibrational adiabaticity since the very opening of the $\nu=1$ entrance channel (which is roughly coincident with the maximum of the collinear $\nu=0$ path). It now becomes clear why we have chosen the top of the $\nu=0$ collinear adiabatic potential to determine the diagonal correction for the BKMP2 PES.

The QCT reaction probabilities have not been represented in this figure, but the classical thresholds can be seen in the excitation functions represented in the two left panels of Fig. 5. The QCT-GB threshold for the reaction with $\mathrm{H}_{2}(\nu=0)$ coincides with its QM counterpart and reflects the already mentioned tendency to preserve the symmetric stretch throughout the reactive collision, in this case reinforced through the Gaussian filter centered at $\mathrm{MuH}(\nu=0)$. The QCT-HB threshold is appreciably lower $(\sim 0.5 \mathrm{eV})$. In the absence of the forcing GB filter the vibrational stretching motion is only partially preserved.

For the reaction of $\mathrm{Mu}$ with $\mathrm{H}_{2}(\nu=1)$ the QCT-HB and QCT-GB threshold is found at $E_{0} \sim 1.05 \mathrm{eV}$, a value clearly higher than that from the QM calculations. The energy of this classical threshold corresponds to that of the first maximum in the $\nu=1$ collinear adiabatic path (left panel of Fig. 6), which implies that the two QCT approaches tend again to preserve the vibrational adiabaticity to a certain extent, in contrast with the QM behaviour just described. For the exoergic $\mathrm{Mu}+\mathrm{H}_{2}$ $(\nu=1) \rightarrow \mathrm{MuH}(\nu=0)+\mathrm{H}$ reaction, the GB filter is not so critical and the preservation of vibrational adiabaticity, which ultimately determines the threshold, is similar in the GB and $\mathrm{HB}$ calculations. This strict preservation of the initial vibrational motion is at the root of the clamorous failure of the QCT calculations to reproduce the rate coefficients of $\mathrm{Mu}+\mathrm{H}_{2}(\nu=1)$, stressed in the discussion of Fig. 1, since the classical calculations cannot account for the tunneling through the $\nu=1$ adiabatic barrier.

Fig. 7 shows the correlation between relevant potential energy paths and reaction probabilities of $\mathrm{D}+\mathrm{H}_{2}$. As in the case of $\mathrm{Mu}$, the threshold for the reaction of $\mathrm{D}$ with $\mathrm{H}_{2}$ $(\nu=0)$ coincides with the maximum of the collinear $\nu=0$ adiabatic path. However for the reaction of deuterium atoms with $\mathrm{H}_{2}(\nu=1)$ the total energy threshold is appreciably higher, which is in contrast with the behaviour observed for the corresponding reaction with $\mathrm{Mu}$. This differing behaviour can be traced back to the fact that in the reaction with $\mathrm{D}$, the HD $(\nu=1)$ exit channel is open at a low energy. In this case, reactants starting on the $\nu=1$ adiabatic path tend to stick to it and, in analogy with the reaction with $\mathrm{H}_{2}(\nu=0)$, the operational QM threshold is given approximately by the maximum in the collinear $\nu=1$ reaction path. Tunneling through the $\nu=1$ collinear adiabatic barrier is not large given the larger relative mass of the $\mathrm{DH}_{2}$ system - and the QCT thresholds, which are again determined by the tendency 


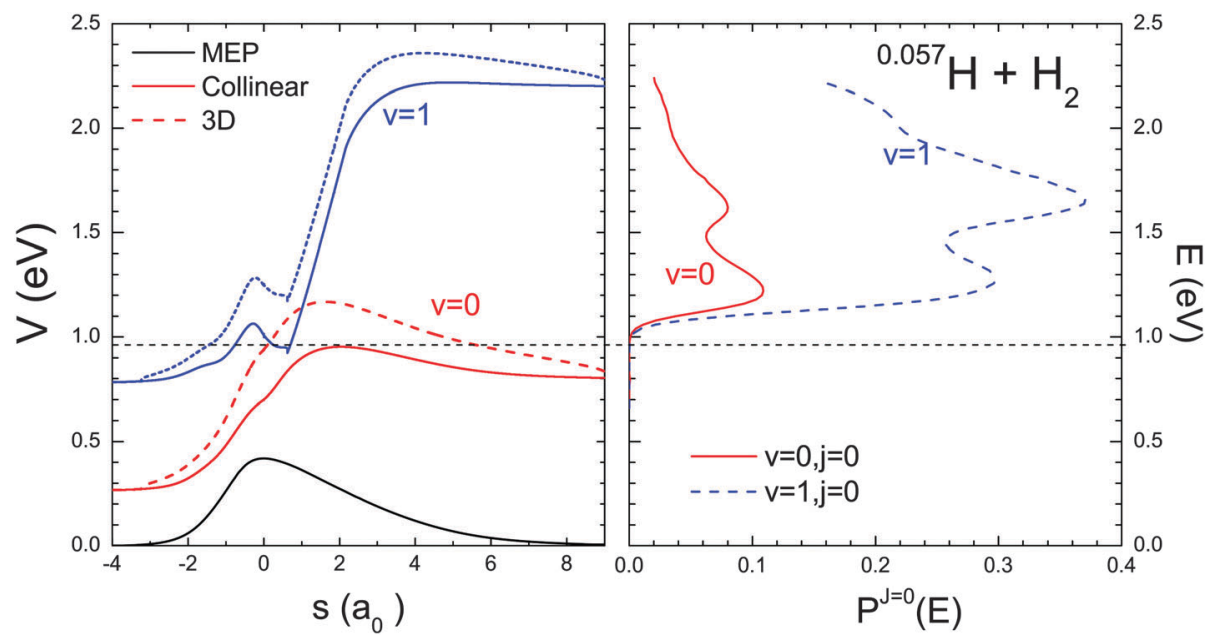

Fig. 8 The same as Fig. 6 but for the reaction of a fictitious isotope with a mass half of that of $\mathrm{Mu}\left({ }^{0.057} \mathrm{H}\right)$.

to preserve the stretching vibrational motion in the course of reactive collisions, lie very close to their operational QM counterparts (see right panels of Fig. 5). Note, however, that although the contribution of $\mathrm{QM}$ tunneling to the global reactivity is comparatively small for $\mathrm{D}+\mathrm{H}_{2}(\nu=1)$, it becomes relevant for the values of the low temperature rate coefficients, as shown in the lower panel of Fig. 2.

To further illustrate the role of tunneling through a vibrationally adiabatic barrier, we have performed calculations for an even lighter fictitious isotope having half the mass of $\mathrm{Mu}$, which we have termed $\mathrm{Mu}(1 / 2)\left({ }^{0.057} \mathrm{H}\right)$, for which a priori more tunneling could be expected. The results of this calculation are represented in Fig. 8. The displayed behaviour is roughly similar to that described for Mu in Fig. 6, but in the case of $\mathrm{Mu}(1 / 2)$, the direct connection between the $\mathrm{QM}$ reaction threshold and the maximum of the collinear adiabatic curve for $\nu=0(0.95 \mathrm{eV})$ is even more clear, since for this isotopic variant the maximum of the $\nu=0$ adiabatic potential is above the opening of the entrance channel for $\nu=1$. The fact that the maximum in the $\nu=0$ collinear adiabatic curve constitutes an "absolute" threshold for each of the isotopic variants and internal states considered supports the conclusions from previous works ${ }^{6,27}$ indicating that the height of the barrier of the adiabatic vibrational stretching curve provides a good transition state ZPE.

\section{Summary and conclusions}

Extensive QM calculations of reaction probabilities, integral cross sections, cumulative reaction probabilities, and rate coefficients have been carried out for the exchange reactions of $\mathrm{Mu}$ and $\mathrm{D}$ with $\mathrm{H}_{2}$ molecules in their ground and first excited vibrational levels aimed to analyze the possible effects of tunneling and the vibrational adiabaticity on the dynamics. QCT calculations have also been performed to compare with the QM data.

The QM rate coefficients for the reactions of the two isotopes with $\mathrm{H}_{2}$ in $\nu=0$ and $\nu=1$ are in excellent agreement with the available experimental results. In particular, QM calculations on the Born-Huang PES and on the BKMP2 PES - in which the adiabatic diagonal correction has been included - predict a rate coefficient for the $\mathrm{Mu}+\mathrm{H}_{2}(\nu=1)$ reaction which is in good agreement with the recent experimental estimate at $300 \mathrm{~K}$. Moreover, the kinetic isotope effect, $k(T ; \nu=1)_{\mathrm{Mu}+\mathrm{H}_{2}} / k(T ; \nu=1)_{\mathrm{D}+\mathrm{H}_{2}}$, for the reactions with $\mathrm{H}_{2}$ in $\nu=1$ predicted by the calculations is within the uncertainty of the measurements.

The huge vibrational enhancement in the $\mathrm{Mu}+\mathrm{H}_{2}$ rate coefficient from $\nu=0$ to $\nu=1$ is indeed remarkable at low temperatures ( 7 orders of magnitude at $300 \mathrm{~K}$ ), much greater than that for $\mathrm{D}+\mathrm{H}_{2}(2$ orders of magnitude at $300 \mathrm{~K})$. More interestingly, the QCT calculations for $\mathrm{Mu}+\mathrm{H}_{2}(\nu=1)$ predict a much smaller $k(T)$ than that obtained with the accurate QM calculations. In contrast to the reaction with $\mathrm{H}_{2}(\nu=0)$, the application of the GB procedure does not improve the agreement with the QM (or experimental) results. This behaviour can only be explained as an effect of tunneling which is practically absent in the reaction with $\mathrm{H}_{2}(\nu=0)$. In the case of the reaction of $\mathrm{D}$ with $\mathrm{H}_{2}$ in $\nu=0$ and $\nu=1$ there is much better agreement between QM and QCT results, as a result of an appreciably smaller contribution of tunneling, only relevant for $T<400 \mathrm{~K}$.

Examination of the QM reaction probabilities and total cross sections shows that the total energy thresholds for the reactions of $\mathrm{Mu}$ with $\mathrm{H}_{2}$ in $\nu=0$ and $\nu=1$ are very similar whereas for the corresponding reaction with $\mathrm{D}$ the $\nu=0$ threshold is about $0.3 \mathrm{eV}$ lower than that for $\nu=1$.

All these results have been rationalized by resorting to the vibrational adiabatic potentials along the minimum energy path. It has been found that the threshold for the reaction of $\mathrm{Mu}$ with $\mathrm{H}_{2}$ in both $\nu=0$ and $\nu=1$ states is practically the same and is given by the height of the $\nu=0$ adiabatic collinear potential (excluding the bending contribution). For the $\mathrm{D}+\mathrm{H}_{2}$ reaction, however, the adiabaticity is preserved and the threshold for the reaction in $\nu=1$ is very close to the height of the $\nu=1$ adiabatic collinear barrier. In the $\mathrm{Mu}+\mathrm{H}_{2}(\nu=1)$ case, where the $\mathrm{MuH}(\nu=1)$ exit channel is closed, there has to be a breakdown of the adiabaticity. At the lowest energies the crossing from the $\nu=1$ to the $\nu=0$ adiabat takes place by tunneling through the relatively small $\nu=1$ barrier. In the classical case such tunneling cannot take place and the threshold coincides with the height of the $\nu=1$ collinear adiabat. 
The support of the Spanish Ministry of Science and Innovation (grants CTQ2008-02578/BQU, FIS2010-16455, and CSD200900038) is gratefully acknowledged. The research was conducted within the Unidad Asociada Química Física Molecular between the UCM and the CSIC of Spain.

\section{References}

1 D. G. Fleming, D. J. Arsenau, O. Sukhorukov, J. H. Brewer, S. L. Mielke, G. C. Schatz, B. C. Garrett, K. A. Peterson and D. G. Truhlar, Science, 2011, 331, 448.

2 P. G. Jambrina, E. García, V. J. Herrero, V. Sáez-Rábanos and F. J. Aoiz, J. Chem. Phys., 2011, 135, 03410.

3 R. Pérez de Tudela, F. J. Aoiz, Y. V. Suleimanov and D. E. Manolopoulos, J. Phys. Chem. Lett., 2012, 3, 493.

4 F. J. Aoiz, L. Bañares and V. J. Herrero, Int. Rev. Phys. Chem., 2005, 24, 119.

5 D. G. Fleming, D. J. Arsenau, O. Sukhorukov, J. H. Brewer, S. L. Mielke, D. G. Truhlar, G. C. Schatz, B. C. Garrett and K. A. Peterson, J. Chem. Phys., 2011, 135, 184310.

6 D. C. Chatfield, R. S. Friedman, D. G. Truhlar and D. W. Schwenke, Faraday Discuss., 1991, 91, 289.

7 D. C. Chatfield, R. S. Friedman, D. W. Schwenke and D. G. Truhlar, J. Phys. Chem., 1992, 96, 2414.

8 L. Bonnet and J.-C. Rayez, Chem. Phys. Lett., 1997, 277, 183.

9 L. Bañares, F. J. Aoiz, P. Honvault, B. Bussery-Honvault and J.-M. Launay, J. Chem. Phys., 2003, 118, 565.

10 F. J. Aoiz, H. Buchenau, V. J. Herrero and V. Sáez-Rábanos, J. Chem. Phys., 1994, 100, 2789.

11 F. J. Aoiz, L. Bañares, V. J. Herrero, V. Sáez-Rábanos and I. Tanarro, J. Phys. Chem. A, 1997, 101, 6165.

12 P. Bakule, O. Sukhorukov, Y. Matsuda, F. Pratt, P. Gumplinger, T. Momose, E. Torikai and D. G. Fleming, Physica B, 2009, 404, 1013.
13 A. I. Boothroyd, W. J. Keogh, P. G. Martin and M. R. Peterson, J. Chem. Phys., 1996, 104, 7139.

14 D. Skouteris, J. F. Castillo and D. E. Manolopoulos, Comput. Phys. Commun., 2000, 133, 128.

15 F. J. Aoiz, M. Brouard, C. Eyles, J. F. Castillo and V. SáezRábanos, J. Chem. Phys., 2006, 125, 144105.

16 F. J. Aoiz, V. J. Herrero, M. P. Miranda and V. Sáez-Rábanos, Phys. Chem. Chem. Phys., 2007, 9, 5367.

17 S. L. Mielke, K. A. Peterson, D. W. Schwenke, B. C. Garrett, D. G. Truhlar, J. V. Michael, M. C. Su and J. W. Sutherland, Phys. Rev. Lett., 2003, 91, 063201.

18 S. L. Mielke, D. W. Schwenke, G. C. Schatz, B. C. Garrett and K. A. Peterson, J. Phys. Chem. A, 2009, 113, 4479.

19 In ref. 5 the authors suggested that $\Delta E_{\mathrm{b}}$ should be estimated at the reaction coordinate of the maximum of the $3 \mathrm{D}$ vibrationally adiabatic potential including the bending contribution. The resulting value for the $\mathrm{Mu}+\mathrm{H}_{2}$ is $\approx 8 \mathrm{meV}$. For reasons that will be discussed hereinafter, we have calcuted $\Delta E_{\mathrm{b}}$ at the maximum of the collinear adiabatic potential.

20 B. C. Garrett, G. C. Lynch, T. C. Allison and D. G. Truhlar, Comput. Phys. Commun., 1998, 109, 47.

21 I. D. Reid, D. M. Garner, L. Y. Lee, M. Senba, D. J. Arsenau and D. G. Fleming, J. Chem. Phys., 1987, 86, 5578

22 K. Tsuda, K. Moribayashi and H. Nakamura, Chem. Phys. Lett., 1994, 231, 439.

23 K. Tsuda, K. Moribayashi and H. Nakamura, J. Chem. Phys., 1995, 103(13), 5512.

24 J. V. Michael, M.-C. Su and J. W. Sutherland, J. Phys. Chem. A, 2004, 108, 432.

25 H. Buchenau, J. P. Toennies, J. Arnold and J. Wolfrum, Ber. Bunsenges. Phys. Chem., 1990, 94, 1231.

26 M. E. Mandy and P. G. Martin, J. Phys. Chem., 1991, 95, 8726.

27 D. C. Chatfield, R. S. Friedman, D. G. Truhlar, B. C. Garrett and D. W. Schwenke, J. Am. Chem. Soc., 1991, 113(2), 486- 494. 